\title{
Mother's own milk dose is associated with decreased time from initiation of feedings to discharge and length of stay in infants with gastroschisis
}

\author{
Andrew P. Storm ${ }^{1} \cdot$ Rakhee M. Bowker ${ }^{2} \cdot$ Samuel C. Klonoski $^{3} \cdot$ Stephanie E. lantorno $^{1} \cdot$ Ami N. Shah $^{3}$. \\ Srikumar Pillai ${ }^{3} \cdot$ Jonathan Bell ${ }^{1} \cdot$ Aloka L. Patel $^{2}$
}

Received: 31 May 2019 / Revised: 20 December 2019 / Accepted: 15 January 2020 / Published online: 28 January 2020

(c) The Author(s), under exclusive licence to Springer Nature America, Inc. 2020

\begin{abstract}
Objective To determine if mother's own milk (MOM) dose after gastroschisis repair is associated with time from feeding initiation to discharge. Secondary outcomes included parenteral nutrition (PN) duration and length of stay (LOS).

Study design Retrospective study of 44 infants with gastroschisis examined demographics, gastroschisis type, PN days, timing of nutrition milestones, feeding composition, and LOS.

Results MOM dose was significantly associated with shorter time to discharge from feeding initiation (adjusted hazard ratio [HR] for discharge per 10\% increase in MOM dose, 1.111; 95\% CI, 1.011-1.220, $p=0.029$ ). MOM dose was also significantly associated with shorter LOS (adjusted HR for discharge per 10\% increase in MOM dose, 1.130; 95\% CI, $1.028-1.242, p=0.011)$.

Conclusions MOM dose was significantly associated with a decrease in time to discharge from feeding initiation and LOS in a dose-dependent manner. Mothers of gastroschisis patients should receive education and proactive lactation support to optimize MOM volume for feedings.
\end{abstract}

\section{Introduction}

Gastroschisis is a congenital abdominal wall defect resulting in the herniation of abdominal contents typically through the right periumbilical anterior abdominal wall into the amniotic cavity, thought to be due to a vascular accident early in embryonic development [1-3]. Worldwide, gastroschisis

Dedication In honor of Sam Klonoski-your warmth, kind nature and love of children will be deeply missed but never forgotten.

This manuscript is original, has not been previously published, and has not been submitted for publication elsewhere while under consideration.

Rakhee M. Bowker

rakhee_bowker@rush.edu

Rush Medical College, Chicago, IL 60612, USA

2 Section of Neonatology, Department of Pediatrics, Rush University Medical Center, Chicago, IL 60612, USA

3 Department of Pediatric Surgery, Rush University Medical Center, Chicago, IL 60612, USA incidence is on the rise. In the US, the incidence of gastroschisis has increased from 2.32 per 10000 births to 4.42 per 10000 births between 1995 and 2005 [4].

Direct exposure of intestines to amniotic fluid for the majority of pregnancy results in variable degrees of intestinal injury, ranging from simple gastroschisis with continuous, uncompromised intestine to complex gastroschisis, characterized by intestinal atresia, necrosis, volvulus, or perforation [1]. Infants with gastroschisis commonly require prolonged parenteral nutrition (PN) due to intestinal ileus and dysmotility, resulting in variable degrees of feeding intolerance [5]. A diagnosis of gastroschisis necessitates surgery and often prolonged postoperative hospital stays. Given the complexity of care and the rising prevalence of gastroschisis, it is imperative to identify interventions that can decrease length of stay (LOS) in the Neonatal Intensive Care Unit (NICU).

The optimal postoperative feeding regimen in infants with gastroschisis is currently unknown, particularly with regard to the role of mother's own milk (MOM) versus formula. Exclusive MOM feedings are recommended for all infants for the first 6 months of life, but formula is generally used when MOM is not available [6]. Numerous studies 
Fig. 1 Study population with exclusions. NICU neonatal intensive care unit, $P I C U$ pediatric intensive care unit.

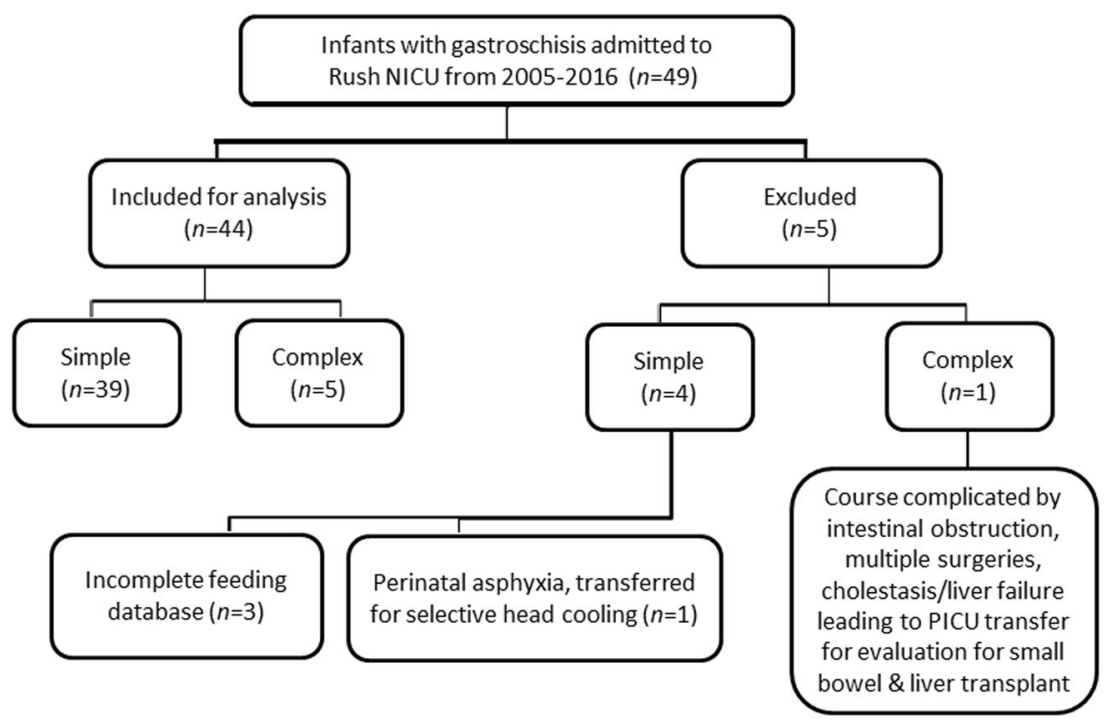

have shown health benefits of MOM over formula for neonates, including improved nutrient absorption, immunological defenses, and maternal-infant bonding [7-11]. The association between MOM use and decreased incidence of necrotizing enterocolitis (NEC) is particularly important in preterm infants, in whom NEC is associated with significant morbidity and mortality [12-14]. However, only a limited literature exists investigating the effect of MOM and/or donor human milk feedings on clinical outcomes in infants with gastroschisis. These studies have demonstrated shorter times to full enteral feedings [15] and shorter time to discharge from initiation of enteral feedings [16] with human milk feedings. Reductions in gastroschisis-related NEC have also been associated with exclusive MOM feedings [17].

The primary objective of this study was to determine the association of MOM dose with time to discharge from initiation of enteral feedings in infants with gastroschisis. Secondary outcomes included PN duration and LOS. We hypothesized that higher doses of MOM would be associated with reduced time from initiation of feedings to discharge, and the secondary outcomes. We utilized time from initiation of feedings to discharge as a surrogate for feeding tolerance and postsurgical complications in these infants, as ability to tolerate enteral feeding advancement is the primary determinant of duration of hospitalization following gastroschisis repair.

\section{Methods}

\section{Study population}

This was a retrospective study of a convenience sample of infants with a diagnosis of gastroschisis admitted to Rush
University Medical Center's NICU from 2005 to 2016. The sole inclusion criterion was admission to the Rush NICU with a diagnosis of gastroschisis during the study years. Forty-nine neonates with gastroschisis met these criteria and were evaluated for inclusion in the study. Five of these 49 were excluded (see Fig. 1). Three infants were excluded due to incomplete database as there was inadequate documentation in the paper medical record regarding composition of daily feedings. One infant was excluded due to concern for perinatal asphyxia resulting in transfer of care soon after birth for selective head cooling. Six infants had complex gastroschisis. One of these infants had intestinal obstruction, underwent multiple surgeries, and was ultimately transferred to the Pediatric Intensive Care Unit with liver failure/cholestasis for evaluation for small bowel/liver transplant. This patient was excluded due to complexity of illness. Due to our relatively small cohort, we chose to include the other five infants with complex gastroschisis, although these complex cases exhibit different clinical courses independent of enteral feeding strategy [18, 19]. The study protocol was reviewed and approved by the Institutional Review Board of Rush University Medical Center and requirement for parental consent was waived.

\section{Study design and data acquisition}

Chart reviews were performed and the following data were abstracted from the medical record: year of birth; gestational age at birth (completed gestational weeks); sex; type of gastroschisis: simple gastroschisis (continuous and uncompromised intestine) versus complex gastroschisis (intestinal atresia, necrosis, volvulus, or perforation) as recorded in the pediatric surgical team's documentation; birth weight, length and occipital frontal circumference (OFC); daily weights; discharge weight, length, and OFC; age in days of 
life (DOL) at surgical closure; age (DOL) at first enteral feeding; age (DOL) at full enteral feedings defined as $140 \mathrm{~mL} / \mathrm{kg} /$ day; PN duration (days); daily volume of each type of enteral nutrition: MOM, Pedialyte since it was occasionally utilized as the first type of postoperative enteral feeding per the pediatric surgeon's discretion, cow's milk based formula, and elemental formula; ventilator days; occurrence of NEC Bell's stage 2 or greater; and LOS. Maternal demographics and method of delivery were also recorded.

The primary outcome time to discharge from the initiation of enteral feedings was calculated by subtracting age in DOL at initiation of feedings from LOS. Time to full enteral nutrition was calculated by subtracting the age at initiation of feedings from age at full enteral feedings. MOM dose was defined as percentage of total enteral nutrition received in the NICU, calculated by dividing total MOM volume by total enteral feeding volume for the infant's entire hospitalization. Of note, donor human milk was not available to gastroschisis infants in the RUMC NICU during the study years. Estimated growth velocity $(\mathrm{g} / \mathrm{kg} /$ day) was calculated using the exponential model as previously described [20].

\section{Statistical analyses}

Descriptive statistics include counts (percentages), mean \pm $\mathrm{SD}$, and median (25th-75th percentile) as appropriate based on distribution. Cox proportional hazards regression modeling was performed to determine the adjusted association between MOM dose as a continuous variable and time from initiation of feeding to discharge. Covariates included in the model were simple versus complex gastroschisis [19], birth gestational age [21], and year of birth to account for any changes in practice over the course of the study period. A secondary analysis was conducted to evaluate two specific cut-points for MOM dose: (1) $>50 \%$ MOM versus $\leq 50 \% \mathrm{MOM}$, and (2) 100\% MOM versus 0-99\% MOM. Survival curves were generated using the Kaplan-Meier method. Cox proportional hazards regression modeling was performed using the same covariates to determine the adjusted association between MOM dose and the secondary outcomes of PN duration and LOS. MOM dose was evaluated as a continuous variable and as the two dichotomous MOM dose categories. All statistical analyses were performed using SPSS ${ }^{\circ}$ Statistics software version 26 (IBM, Armonk, NY). Significance was set at $\alpha<0.05$.

\section{Results}

A total of 44 infants were included in the analysis, of which five infants were classified as complex cases (Fig. 1).
Table 1 Birth data, sociodemographic characteristics, and clinical course.

\begin{tabular}{|c|c|}
\hline Infants with gastroschisis $(n=44)$ & $\begin{array}{l}\text { Mean } \pm \mathrm{SD} \text {, median } \\
\text { [interquartile range] or } N(\%)\end{array}$ \\
\hline \multicolumn{2}{|l|}{ Birth characteristics } \\
\hline Birth weight (g) & $2189 \pm 577$ \\
\hline Birth length $(\mathrm{cm})(n=42)$ & $43.6 \pm 5.1$ \\
\hline Birth FOC $(\mathrm{cm})(n=42)$ & $30.7 \pm 2.1$ \\
\hline Birth gestational age (weeks) ${ }^{\mathrm{a}}$ & $35.7(33.9,37.1)$ \\
\hline Male & $23(52 \%)$ \\
\hline Caesarean section & $25(57 \%)$ \\
\hline Inborn & $42(96 \%)$ \\
\hline Maternal age (years) & $20.9 \pm 3.8$ \\
\hline \multicolumn{2}{|l|}{ Race/Ethnicity } \\
\hline White & $12(27 \%)$ \\
\hline Black & $13(30 \%)$ \\
\hline Hispanic & $18(41 \%)$ \\
\hline Native American & $1(2 \%)$ \\
\hline \multicolumn{2}{|l|}{ Apgar score ${ }^{\mathrm{a}}$} \\
\hline $1 \mathrm{~min}$ & $8(6,9)$ \\
\hline $5 \mathrm{~min}$ & $9(8,9)$ \\
\hline \multicolumn{2}{|l|}{ Gastroschisis type } \\
\hline Simple & $39(89 \%)$ \\
\hline Complex & $5(11 \%)$ \\
\hline \multicolumn{2}{|l|}{ Feeding characteristics } \\
\hline Exclusively MOM-fed infants & $7(16 \%)$ \\
\hline Exclusively formula-fed infants & $1(2 \%)$ \\
\hline Received any hydrolyzed formula & $17(39 \%)$ \\
\hline Average daily MOM dose $>50 \mathrm{~mL} / \mathrm{kg} /$ day & $22(50 \%)$ \\
\hline MOM dose $>50 \%$ enteral intake & $25(57 \%)$ \\
\hline \multicolumn{2}{|l|}{ Nicu course } \\
\hline Ventilator duration (days) $^{\mathrm{a}}$ & $4.5(1,8)$ \\
\hline Course complicated by NEC & $2(4 \%)$ \\
\hline Age at surgical closure $(\mathrm{DOL})^{\mathrm{a}}$ & $6(4,9)$ \\
\hline Never fed & $0(0 \%)$ \\
\hline Never received full enteral feedings & $3(7 \%)$ \\
\hline PN duration (days) ${ }^{\mathrm{a}}$ & $27.5(20.3,87.5)$ \\
\hline Age at start of enteral feeding $(\mathrm{DOL})^{\mathrm{a}}$ & $17(12,23.5)$ \\
\hline Age at full feeds $(\mathrm{DOL})^{\mathrm{a}}(n=41)$ & $29(23,38)$ \\
\hline $\begin{array}{l}\text { Time from feeding initiation to full feeds } \\
(\text { days })^{\mathrm{a}}(n=41)\end{array}$ & $12.5(8,21.3)$ \\
\hline $\begin{array}{l}\text { Time from feeding initiation to discharge } \\
\text { (days) }^{\mathrm{a}}\end{array}$ & $24(15,40.5)$ \\
\hline Length of NICU Stay (days) ${ }^{\mathrm{a}}$ & $41.5(30.3,60.5)$ \\
\hline Post menstrual age at discharge (weeks) ${ }^{\mathrm{a}}$ & $41.3(39.8,43.7)$ \\
\hline Discharge weight (g) & $3322 \pm 1121$ \\
\hline Discharge length $(\mathrm{cm})(n=43)$ & $49.4 \pm 4.7$ \\
\hline Discharge FOC $(\mathrm{cm})(n=32)$ & $35.1 \pm 2.2$ \\
\hline $\begin{array}{l}\text { Estimated growth velocity in NICU }(\mathrm{g} / \mathrm{kg} / \\
\text { day) }\end{array}$ & $7.4 \pm 2.7$ \\
\hline
\end{tabular}

${ }^{\mathrm{a}}$ Presented as median (25th percentile, 75 th percentile).

$D O L$ days of life, FOC fronto-occipital circumference, $L O S$ length of stay, MOM mother's own milk, NICU neonatal intensive care Unit, $P N$ parenteral nutrition.

Infants were evenly distributed across the study years. Cohort characteristics, feeding milestones, and NICU hospital course details are reported in Table 1. 


\section{Primary outcome}

Cox proportional hazards regression modeling adjusting for gestational age, gastroschisis type, and year of birth demonstrated that MOM dose was significantly associated with a shorter time to discharge from initiation of enteral feedings (adjusted hazard ratio [HR] for discharge per $10 \%$ increase in MOM dose, $1.111 ; 95 \% \mathrm{CI}$, $1.011-1.220, p=0.029)$. Additional analyses demonstrated that MOM dose dichotomized at $>50 \%$ of enteral intake was not significantly associated with the primary outcome. However, $100 \%$ or exclusive MOM dose was significantly associated with a shorter time to discharge from the initiation of enteral feedings compared with lower $(0-99 \%$ MOM) doses (adjusted HR for discharge, 3.888 ; 95\% CI, $1.406-10.751, p=0.009$ ) as displayed in Fig. 2.

\section{Secondary outcomes}

MOM dose was not associated with PN days, regardless of MOM dose method utilized (continuous, $>50 \%$ or exclusive). MOM dose was significantly associated with a shorter LOS (adjusted HR for discharge per 10\% increase in MOM dose, $1.130 ; 95 \%$ CI, 1.028-1.242, $p=0.011)$. MOM dose dichotomized at $>50 \%$ of enteral intake was not significantly associated with LOS. However, $100 \%$ or exclusive MOM dose was also significantly associated with a shorter LOS compared with lower (0-99\% MOM) doses (adjusted HR for discharge, 6.365; 95\% CI, 2.072-19.547, $p=0.001)$.

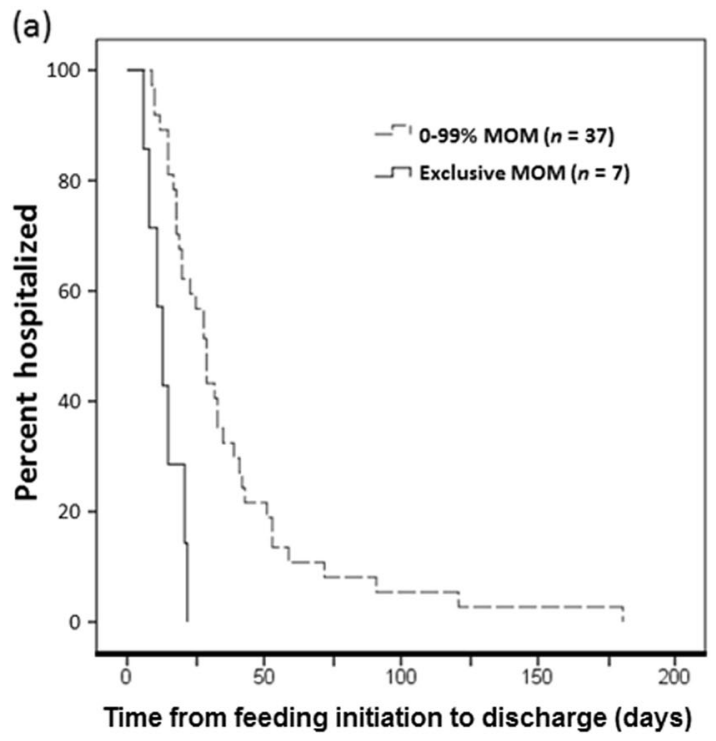

Fig. 2 Exclusive MOM feedings were associated with (a) shorter time to discharge from initiation of enteral feedings, and (b) shorter LOS when compared with $0-99 \%$ MOM feedings for infants admitted to RUMC with gastroschisis. Kaplan-Meier curves are presented with

\section{Discussion}

In this study, we found that MOM dose was significantly associated with a decrease in time to discharge from the initiation of enteral feedings and LOS. Our findings demonstrate a dose-dependent effect of MOM which was identified using daily enteral feeding volumes to accurately calculate MOM dose.

Previous studies have examined the relationship between feeding type and clinical outcomes in infants with gastroschisis and other abdominal wall defects and have reported similar outcomes, although the methodology has varied among studies [15, 16, 22]. Kohler et al. studied 79 infants with simple gastroschisis admitted prior to the institution's use of donor human milk [23] and divided them into four groups: exclusive MOM, 50-99\% MOM, 1-49\% MOM, and all cow's milk based formula. The investigators found that exclusively MOM-fed infants had a shorter time from initiation of feedings to hospital discharge compared with the other groups [15]. They also reported a significant association between MOM dose as a continuous variable and time from the initiation of feedings to hospital discharge and LOS; however, they did not report hazard ratios.

These findings were corroborated in a 2016 study of 3082 patients with gastroschisis admitted to 300 NICUs included in the Pediatrix Medical Group Clinical Data Warehouse [16]. Human milk intake (defined as MOM or donor human milk) was determined based on the proportion of days infants received human milk of the days they received any enteral feedings. Gulack et al. found a clear benefit from being fed human milk on $100 \%$ of fed days or

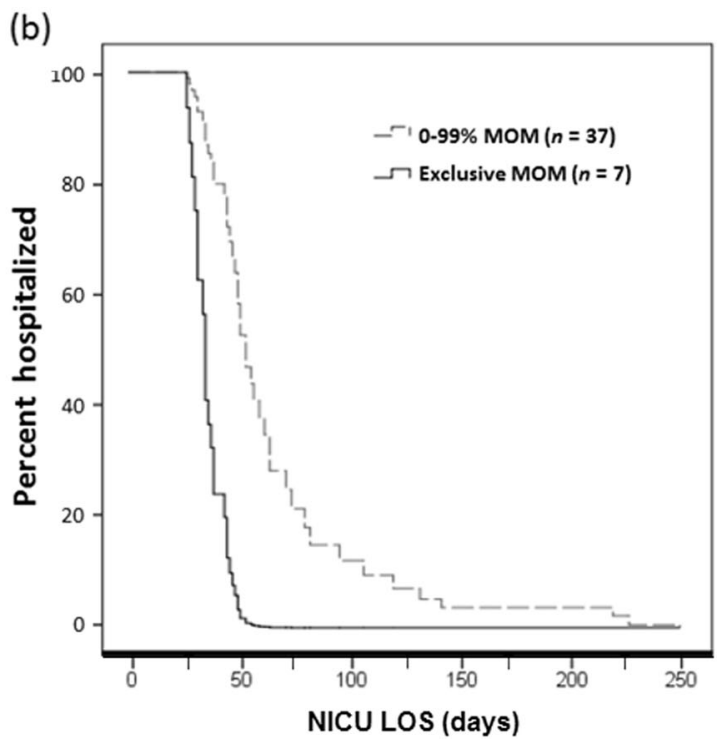

percent of infants hospitalized on the $Y$-axis and time from feeding initiation to discharge (a) and LOS (b) on the $X$-axis. LOS length of stay, $M O M$ mother's own milk. 
on $51-99 \%$ of fed days with a shorter time from initiation of feedings to hospital discharge compared with receiving no human milk. Interestingly, they found that receiving human milk on $1-50 \%$ of feeding days was associated with a longer time from initiation of feeds to discharge than being fed $0 \%$ human milk, which the authors attributed to incomplete adjustment for severity of illness. Specifically, they were unable to adjust for complexity of gastroschisis, which has been shown to impact outcomes in infants with gastroschisis [21].

Our results highlight the importance of lactation support for mothers of infants with surgical conditions in the NICU, as not all mothers have sufficient milk supply to provide exclusive MOM feedings. Given that the infants with gastroschisis do not receive enteral feedings immediately after birth and are often maintained nil per os for several weeks, their mothers must rely on breast pumps to initiate and maintain lactation. Recent qualitative research demonstrates that these mothers report suboptimal lactation education and support [24]. Many NICUs have developed strategies to improve lactation care for mothers of premature infants. It is equally important to address the unique needs of mothers of infants with surgical conditions.

Currently our NICU limits use of donor human milk to very low birth weight infants (birth weight $<1500 \mathrm{~g}$ ) and preterm infants born at $<32$ weeks gestational age for feeding in lieu of formula when MOM is not available. To our knowledge, no randomized controlled trial of donor human milk feedings in infants with gastroschisis has been published, although clinical trials have been completed and are in progress.

This study has several limitations; most importantly that it is a retrospective review in a single center with a relatively small sample size. While we attempted to adjust for several important covariates, we were not able to account for other potentially important covariates such as sociodemographic factors [25] due to the sample size. Strengths of our study included the detailed MOM dose measures, inclusion of covariates that have been identified as predictors of outcomes in infants with gastroschisis, and inclusion of birth year in analyses to adjust for variation in practice that may have occurred over the 12 year span of the study.

Combining our data that has specifically examined MOM feedings with the existing literature examining human milk suggests improved clinical outcomes for infants with gastroschisis receiving human milk as opposed to formula, suggesting there may be a role for utilizing donor human milk instead of formula when MOM is not available. These preliminary findings are provocative and support randomized trials examining the role for donor human milk when MOM is unavailable in the postoperative feeding regimen for infants with gastroschisis.
Acknowledgements We would like to thank Dr Michael Schoeny (Rush University Medical Center) for his statistical support. This study was funded by the Rush University Medical Center Dean's Summer Research Fellowship.

Funding This study was funded by the Rush University Medical Center Dean's Summer Research Fellowship.

Author contributions All authors contributed to the study and approved the final manuscript. Conceptualization, AS, JB, and ALP; methodology, AS, RMB, JB, and ALP; data curation, AS, JB, SK, and SI; formal analysis, RMB and ALP; writing-original draft preparation, AS and RMB; writing - review and editing, SK, SI, ANS, SP, JB, and ALP; supervision, RMB, ANS, SP, and ALP; funding acquisition, $\mathrm{AS}$ and ALP.

\section{Compliance with ethical standards}

Conflict of interest The authors declare that they have no conflict of interest.

Publisher's note Springer Nature remains neutral with regard to jurisdictional claims in published maps and institutional affiliations.

\section{References}

1. Lubinsky M. Hypothesis: estrogen related thrombosis explains the pathogenesis and epidemiology of gastroschisis. Am J Med Genet A. $2012 ; 158: 808-11$.

2. Lubinsky M. A vascular and thrombotic model of gastroschisis. Am J Med Genet A. 2014;164A:915-7.

3. Raveenthiran V. Etiology of gastroschisis. J Neonatal Surg. 2012; 1:53.

4. Kirby R, Marshall J, Tanner J, et al. Prevalence and correlates of gastroschisis in 15 states, 1995 to 2005. Obstet Gynecol. 2013;122 (2 Pt 1):275-81.

5. Lao OB, Larison C, Garrison MM, Waldhausen JHT, Goldin AB. Outcomes in neonates with gastroschisis in US children's hospitals. Am J Perinatol. 2010;27:97-102.

6. American Academy of Pediatrics. Breastfeeding and the use of human milk. Pediatrics. 2012;129:e827-41.

7. Andres A, Cleves MA, Bellando JB, Pivik R, Casey PH, Badger TM. Developmental status of 1-year-old infants fed human milk, cow's milk formula, or soy formula. Pediatrics. 2012;129:1134-40.

8. Walker A. Human milk as the gold standard for protective nutrients. J Pediatr. 2010;156:S3-S7.

9. Narayanan I, Prakash K, Bala S, Verma RK, Gujral VV. Partial supplementation with expressed breast-milk for prevention of infection in low-birth-weight infants. Lancet. 1980;2: $561-3$.

10. McMillan JA, Oski FA, Lourie G, Tomarelli R, Landaw SA. Iron absorption from human milk, simulated human milk, and proprietary formulas. Pediatrics. 1977;60:896-900.

11. Feldman R, Eidelman AI. Direct and indirect effects of human milk on the neurobehavioral and cognitive development of premature infants. Dev Psychobiol. 2003;43:109-19.

12. Johnson TJ, Patel AL, Bigger HR, Engstrom JL, Meier PP. Cost savings of human milk as a strategy to reduce the incidence of necrotizing enterocolitis in very low birth weight infants. Neonatology. 2015;107:271-6. 
13. Meinzen-Derr J, Poindexter B, Wrage L, Morrow AL, Stoll B, Donovan EF. Role of human milk in extremely low birth weight infants' risk of necrotizing enterocolitis or death. J Perinatol. 2009;29:57-62.

14. Lucas A, Cole TJ. Human milk and neonatal necrotising enterocolitis. Lancet. 1990;336:1519-23.

15. Kohler J, Perkins A, Bass A. Human milk versus formula after gastroschisis repair: effects on time to full feeds and time to discharge. J Perinatol. 2013;33:627-30.

16. Gulack B, Laughon M, Clark R, et al. Enteral feeding with human milk decreases time to discharge in infants following gastroschisis repair. J Pediatr. 2016;170:85-9.

17. Jayanthi S, Seymour P, Puntis JWL, Stringer MD. Necrotizing enterocolitis after gastroschisis repair: a preventable complication? J Pediatr Surg. 1998;33:705-7.

18. Arnold M, Chang DC, Nabaweesi R, Colombani PM, Bathurst MA, Mon KS, et al. Risk stratification of 4344 patients with gastroschisis into simple and complex categories. J Pediatr Surg. 2007;42:1520-5.

19. Melov SJ, Tsang I, Cohen R, Badawi N, Walker K, Soundappan SSV, et al. Complexity of gastroschisis predicts outcome: epidemiology and experience in an Australian tertiary centre. BMC Pregnancy Childbirth. 2018;18:222.

20. Patel AL, Engstrom JL, Meier PP, Jegier BJ, Kimura RE. Calculating postnatal growth velocity in very low birth weight (VLBW) premature infants. J Perinatol. 2009;29:618-22.

21. Overcash RT, DeUgarte DA, Stephenson ML, Gutkin RM, Norton ME, Parmar S, et al. Factors associated with gastroschisis outcomes. Obstet Gynecol. 2014;124:551-7.

22. Shinnick JK, Wang E, Hulbert C, et al. Effects of a breast milk diet on enteral feeding outcomes of neonates with gastrointestinal disorders. Breastfeed Med. 2016;11:286-92.

23. Brenner M. "Got milk?" development of a children's hospitalbased donor human milk bank: king's daughters milk bank at CHKD. Breastfeed Med. 2015;0:1-2.

24. Demrici J, Caplan E, Brozanski B, Bogen D. Winging it: maternal perspectives and experiences of breastfeeding newborns with complex congenital surgical anomalies. J Perinatol. 2017;38:708-17.

25. Anstey EH, Chen J, Elam-Evans LD, Perrine CG. Racial and geographic differences in breastfeeding-United States, 20112015. Morb Mortal Wkly Rep. 2017;66:723-7. 